\section{CONGRESS COURSE ABSTRACTS}

\section{EFLM Course: How to write a good scientific and professional article?}

Sverre Sandberg, Noklus, Norway - Elvar Theodorsson, Ike/Klinisk Kemi, Sweden

Introduction

Scientific manuscripts can be written in solitude, but it is commonly more productive and more likely to result in better quality if several persons collaborate. The present course builds on this premise and on the understanding that mentored learning through an intensive work of the participants themselves is the most effective method of learning.

The present 48 hours EFLM course in the writing of scientific manuscripts rests on four pillars:

The task of turning a scientific study with resulting data that the participants get from the outset into a manuscript written in English as when intended for publication in an international scientific journal

The participants work closely together in groups of 5-8 persons chosen by the mentors. The group work is performed in the participants own mother tongue whereas the communication of the group with the mentors and in the written parts of manuscript will be in English.

The individual participants are expected to take and maintain the initiative during the entire course in creating and maintaining a fruitful working relation in the groups and in utilizing their own knowledge and skills and those of the two mentors optimally for finishing the task of the group in a fruitful and timely manner.

The two mentors will have brief introductory lectures and subsequently rotate amongst the groups for advice on request of the members of the groups and for making short (about $20 \mathrm{~min}$ ) presentations.

The tasks of the participants

Maintaining an open mind regarding the knowledge and skills of all other members of the group

Contributing all the relevant knowledge and skills of the participants towards the common goals of the group

Introductory lectures of about 20 minutes each

Day 1 The study and data that makeup the data for the current manuscript

- The Material and methods part of the manuscript

- The Results part of the manuscript

- The Introduction part of the manuscript

Day 2 The Discussion part of the manuscript

- The Title, index words and Abstract parts of the manuscrip

\section{miRNA isolation and expression training program}

Aylin Sepici Dincel, Gazi University, Turkey - Oytun Portakal, Hacettepe University, Turkey

miRNAs regulate various metabolic and cellular pathways that specifically control cell proliferation, migration, differentiation and signal transduction by negatively affecting the expression of target mRNAs. Numerous microRNAs have been identified that play a role in the transcription and translation of tumor suppressor genes or oncogenes in many solid tumors and hematologic malignancies. The relationship between microRNA expression levels and metastasis and survival in cancer patients was investigated. Since microRNAs have tissue-specific expression profiles and effective and reliable methods of expression quantification can be performed, microRNA-based markers are important in cancer diagnosis, prognosis and follow-up of treatment. In addition, microRNAs are involved in the regulation of radiotherapy and chemotherapy responses of tissues.

Objective

To gain knowledge and ability to detect and evaluate the amount of miRNA expression from different samples. The theoretical part of the course will provide information on miRNA isolation methods and details, methods for determining the amount of miRNA, amplification methods and how to normalize them. For the isolation of miRNA, the methods of isolation from classical tissue or cell culture as well as direct isolation from plasma will be discussed and their effectiveness will be examined. Sources of errors and strategies to avoid mistakes will be explained. In the application part, suitable samples prepared for miRNA isolation will be used and individual study will be provided. The quantification and expression of miRNAs obtained will be discussed with visual materials prepared by real-time PCR method. Information will be given about normalization. Clinical evaluation of identified up-regulated and down-regulated miRNA profiles will be performed.

Learning Objective

To be able to create a different view of these molecules with the recent developments in the field of research of miRNAs that attract attention with biomarker studies.

At the end of this course participants will:

1. explain and apply miRNA isolation methods from plasma and different tissues 2. be able to quantify miRNA

3. identify miRNA expression profiles

4. apply normalization, basic clinical evaluation issues

Target group

Researchers and postgraduate students and specialists in biochemistry.

Necessary knowledge and skills

Molecular genomic techniques should have a basic level of knowledge.

\section{Applying Six Sigma to analytical performance in the medical laboratories}

Hassan Bayat, Iran

\section{Introduction}

$14: 45-15: 15$

The concepts of Sigma Metric

The history of the development of this metric

Definitions of Acceptable Product, Acceptable Performance, the meaning of Defects per Million Opportunities (DPMO)

Two Approaches to Calculating Sigma-metric

15:15-16:00

The counting approach vs. statistical approach to determine defect rate Statistical approach is appropriate for estimating error rate of analytical phase Sigma-metric in Evaluation of Analytical Performance

16:00-16:45

The equation to determine the analytical Sigma-metric

The components necessary to calculate Sigma-metric: TEa, bias and imprecision Sigma-metric in QC Planning

$16: 45-17: 45$

The relation between Sigma and patient risk

Characteristics of statistical quality control

Adjusting QC based on Sigma: QC rules, number of QC assays per event, and $\mathrm{QC}$ frequency

$\mathrm{Q} \& \mathrm{~A}$

$17: 45-18: 15$

The audience will present their comments and questions

\section{Mass Spectrometer use in clinical laboratory practice (Basic Course)}

Prof. Dr. Ali Ünlü, Selçuk University, Konya

Prof. Dr. Muhittin Serdar, Acıbadem University, İstanbul

Doç. Dr. Sedat Abuşoğlu, Selçuk University, Konya

AIM: Mass spectrometric techniques are increasingly used in clinical laboratory. The aim of this course is to reach a sufficient level of knowledge about working principles, procedures, aspects to be considered and application areas of mass spectrometry.

COURSE OUTLINE:

Liquid chromatography principles,

Mass Spectrometer (MS) techniques

Setting up a clinical mass spectrometer lab, calibrator and reagent selection in MS methods, quantitative result evaluation.

MS applications in clinical laboratories, comparison with immunassays.

Method validation and verification process in MS

Discussion

PARTICIPANT PORTFOLIO:

Medical biochemistry specialists, $\mathrm{PhD}$, research assistants, graduate students, device users. 
XXVII. Balkan Clinical Laboratory Federation Meeting BCLF 2019

XXX. National Congress of the Turkish Biochemical Society TBS 2019

\section{Mass spectrometer use in clinical laboratory practice (Advance Course)}

Prof. Dr. Ali Ünlü, Selçuk University, Konya

Prof. Dr. Muhittin Serdar, Acıbadem University, İstanbul

Doç. Dr. Sedat Abuşoğlu, Selçuk University, Konya

AIM: Advanced mass spectrometer training course for partially experienced specialists who have mass spectrometer in their laboratory and familiar with mass spectrometer use.

COURSE OUTLINE:

"In house "method development in mass spectrometer laboratories and verification of commercial kits (CLSI 62-A)

Maintenance of mass spectrometry and troubleshooting, maintenance of quality in mass spectrometry analysis

Use of MS in metabolism laboratories

Method selection in analytical toxicology

Mass spectrometry use in endocrine laboratories

Discussion

PARTICIPANT PORTFOLIO:

Medical biochemistry specialists, $\mathrm{PhD}$, research assistants, graduate students, device users. 